\title{
Iterative Learning Based Modulating Functions Method for Distributed Solar Source Estimation
}

\author{
Fahad Aljehani, Taous-Meriem Laleg-Kirati, IEEE Senior Member
}

\begin{abstract}
Modulating functions method is a non asymptotic estimation method, which provides accurate and robust estimations of states, parameters and inputs for different classes of systems, which include unknown linear ordinary differential systems, fractional systems and linear partial differential equations. In the case of time or space varying unknown, the method requires the decomposition of the unknown into predefined basis functions. However, the estimation performance will depend on the nature of the basis functions which in some cases are not easy to determine. This paper proposes a new iterative learning based modulating functions method, which combines the standard modulating functions with a dictionary learning procedure. The dictionary learning step allows the determination of appropriate set of functions to decompose the unknown, while the modulating function step allows the non-asymptotic and robust estimation of the projection coefficients. The performance of the proposed method is illustrated in a distributed solar collector application, modeled by partial differential equations and where the unknown solar irradiance is estimated.
\end{abstract}

Index Terms-Dictionary learning, modulating functions, non-asymptotic estimation.

\section{INTRODUCTION}

$\mathbf{I}$ $\mathrm{N}$ general, complex dynamic systems are commonly represented by partial differential equation (PDE). An example of such a system, which is considered in this paper, is a distributed solar collector where the source term refers to energy efficiency. Various studies have focused on designing control strategies to enhance the heat production of the distributed solar collector by tuning the flow rate to track a desired reference [8], [13], [22]. Depending on the solar irradiance and mirrors optical efficiency, which are usually considered as disturbances, the dynamics and heat production will be affected accordingly. On the other hand, using pyrheliometer, which produces local measurements of the solar irradiance, might lead to inaccurate measurements when the solar field is large [12]. Therefore, there is a potential need to estimate the solar irradiance source term.

Various estimation techniques have been proposed to estimate the parameters or source term for PDE systems. One

This work has been supported by the King Abdullah University of Science and Technology (KAUST), Base Research Fund (BAS/1/162701-01) to Taous Meriem Laleg.

Fahad Aljehani and Taous-Meriem Laleg-Kirati Computer, Electrical and Mathematical Sciences and Engineering Division (CEMSE), King Abdullah University of Science and Technology (KAUST), Thuwal 23955-6900, Saudi Arabia fahad.aljehani@kaust.edu.sa taousmeriem.laleg@kaust.edu.sa approach is based on optimization which is focusing on minimizing a proper choice of the cost function with respect to all the unknown parameters [17]. Along with the computational complexity, this approach depends heavily on the stopping criteria and initial guess to obtain accurate estimation. Another estimation approach consists in recursive algorithms such as observers [5]. This approach is restricted to satisfy the conditions of observability and detectability which may be lost during the numerical approximation of the PDE. Whereas, most of the recursive algorithms target asymptotic convergence which is not suitable for control objectives.

Modulating functions method (MFM) has been developed in the fifties by Shinbrot for the estimation of constant parameters of ordinary differential equations [21]. Later on, the method has been extended to solving estimation problems for PDEs of constant and space varying parameters by Perdreauville and Goodson [19]. And more recently, it has been studied both mathematically and numerically for solving state, parameters and input estimation problems for different classes of systems, including fractional systems and PDEs [14]-[16]. In particular, MFM has been used to estimate in-domain source term of the solar collectors whereas the PDE model of the source term includes solar irradiance intensity and properties of the collectors [11]. Nevertheless, MFM has been investigated to fault diagnosis for some classes of distributed parameters systems [10]. In addition, an online estimation strategy based on MF has been proposed where the time interval is subdivided into time sliding windows where the estimation problem is solved [2]. Whereas, an algorithm for selecting the modulating functions based on the measurements supports has been proposed in [4]. Using predefined basis functions can not be generalized to all applications. Indeed, it is not easy to determine the nature of the basis functions that results in good estimation [6], [10]. For more details about the modulating functions, the reader can refer to [20] and [3].

In case of time or space varying unknown, the method requires the decomposition of the unknown into predefined basis functions. However, the estimation performance will depend on the nature of the basis functions which in some cases not easy to determine. Furthermore, the performance analysis with respect to basis functions has not been thoroughly investigated, to the best of our knowledge. In fact, the selection of predefined basis functions can affect the estimation results. In this work, an iterative learning based modulating functions method is proposed for adaptive estimation of the source term of the distributed solar collector model. The present paper contains the following contributions. 
- A new iterative learning algorithm that determine the basis function framework in the source estimation problem is proposed. The algorithm combines the standard modulating functions with a dictionary learning procedure to enhance the estimation performance results.

- The effectiveness of the proposed algorithm is demonstrated in a distributed solar collector system.

- The proposed iterative estimation algorithm based on both modulating functions method and dictionary learning (MFM-DL) can be extended to a large class of systems.

The outline of the paper is organized as follows: In Section II, we describe the solar collector system. In Section III, the main results of the estimation problem based on both the modulating function method and dictionary learning approach (MFM-DL) are derived. Numerical simulation tests of the proposed iterative learning based on modulating functions method, including synthetic and real solar irradiance profiles, are presented. Finally, concluding remarks of the proposed method (MFM-DL) are presented in Section V.

\section{Distributed Solar Collector Plant}

The heat transfer in a concentrated distributed solar collector is described by a first order hyperbolic PDE. It is obtained by neglecting the thermal exchange and the heat losses between the thermal carrier and the collector tube, as illustrated in Fig 1.

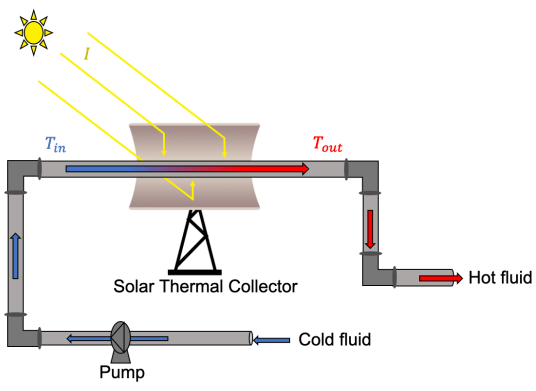

Fig. 1. Parabolic solar collector plant.

Based on an energy balance analysis, the dynamics of the process is given by [7]:

$$
\left\{\begin{array}{l}
\frac{\partial T(x, t)}{\partial t}+u(t) \frac{\partial T(x, t)}{\partial x}=S(t), x \in(0, L], t \in \mathbb{R}^{+} \\
T(0, t)=T_{\text {in }}(t), \quad t \in \mathbb{R}^{+} \\
T(L, t)=T_{\text {out }}(t)=y(t), \quad t \in \mathbb{R}^{+}
\end{array}\right.
$$

where $x$ refers to the position along the solar collector tube, $t$ to the time instant, and $L$ to the length of the tube. $T(x, t)$ refers to the temperature fluid at position $x$ along the tube at time $t$. The source term is defined by $S(t)=\frac{\nu G}{\rho c A_{s}} I(t)$ whereas the effective source term $S(t)$ depends on the solar irradiance $I(t)$. The fluid velocity is defined by $u(t)=\frac{Q(t)}{A_{s}}$ whereas $u(t)$ depends on the pump volumetric flow rate $Q(t)$. The parameters of the system is summarized in Table I. The objective is to estimate the source term $S(t)$ by boundary measurements $T(L, t)$ and $\left.\frac{\partial}{\partial x} T(x, t)\right|_{x=L}$. Depending on the pump volumetric flow rate $Q(t)$, the boundary measurements will be obtained accordingly.

TABLE I

Solar Collector Parameters [7]

\begin{tabular}{|c|c|c|}
\hline Symbol & Unit & Description \\
\hline$t$ & $\mathrm{~s}$ & Time \\
$x$ & $\mathrm{~m}$ & space \\
$T(x, t)$ & ${ }^{\circ} \mathrm{C}$ & temperature \\
$Q(t)$ & $\mathrm{m}^{3} \mathrm{~s}^{-1}$ & Volumetric fluid flow rate \\
$\rho$ & $\mathrm{kg} \mathrm{m}^{-3}$ & Fluid density \\
$c$ & $\mathrm{~J}^{\circ} \mathrm{C}^{-1} \mathrm{~kg}^{-1}$ & Fluid specific heat capacity \\
$A_{s}$ & $\mathrm{~m}^{2}$ & Cross-sectional area \\
$\nu$ & & Mirror optical efficiency \\
$G$ & $\mathrm{~m}$ & Mirror optical aperture \\
$I(t)$ & $\mathrm{W} \mathrm{m}$ & Solar irradiance \\
$L$ & $\mathrm{~m}$ & Tube length \\
\hline
\end{tabular}

\section{ITERATIVE LEARNING BASED ON MODULATING FUNCTIONS METHOD}

This work aims to develop an adaptive algorithm to obtain a good estimation of the source term by the outlet temperature measurements. The estimation of the source term is useful since it is usually unknown and considered as a disturbance. An accurate estimation of the source term helps to understand the underlying dynamics of the solar collector system and how heat production can be enhanced. Moreover, modulating function method avoids taking derivative of noisy measurement which helps to prevent numerical instability. In addition to that, the estimation problem can be reformulated to algebraic system instead of solving the PDE system. However, previous results on the modulating function approach lack strong analysis of a suitable basis function, which might affect the estimator's convergence.

In this study, we propose to use a dictionary learning procedure to enhance the performance of the MFM. Dictionary learning algorithm decomposes or projects any signal into basis functions and basis coefficients. It has been used to have sparse representations of the basis coefficients. Meaning, the signal can be reconstructed with a few elements of the basis coefficients. The objective of the training procedure of dictionary learning algorithm is to conclude with updated basis functions.

To this end, solving the basis coefficients during the training process of updating the basis function requires the complete knowledge of the original signal which is usually not considered as estimation. Hence, we propose an adaptive estimation algorithm that combines a non-asymptotic estimation and dictionary learning framework called MFM-DL to estimate the source term.

\section{A. Modulating Functions Methods}

Modulating functions method is used as parameters estimation algorithms where it transforms the estimation problem into an algebraic linear system. It also transfers the derivative from noisy measurements to the known modulating functions that we designed to be smooth enough. As the MFM has been developed for constant unknowns, the estimation of a 
time varying source requires the decomposition of the source into a set of known basis functions and then to estimate the unknown projection coefficients. The process begins by decomposing the source term of the solar collector into known basis functions as follows:

$$
S(t)=\sum_{k=1}^{K} \alpha_{k} \bar{d}_{k}(t)
$$

where $\alpha_{k}$ is the basis coefficients, $\bar{d}_{k}(t)$ is the basis functions, and $K$ is the number of basis. Although, the choice of the basis functions is crucial and actually can lead to inaccurate estimations.

Before starting with the procedure of modulating functions method, let define a modulating function.

Definition 1: [21] A function $\phi(t) \neq 0$ is called modulating function of order $l\left(l \in \mathbb{N}^{\star}\right)$ on $\left[t_{0}, t_{f}\right]$ if the two properties below are satisfied

$$
\left\{\begin{array}{l}
\phi(t) \in \mathcal{C}^{l}\left(\left[t_{0}, t_{f}\right]\right) \\
\phi^{(p)}\left(t_{0}\right)=\phi^{(p)}\left(t_{f}\right)=0 \quad p=0,1, \ldots, l-1,
\end{array}\right.
$$

where $t_{f}>t_{0}$ and $p$ is the derivative order. The procedure for parameters estimation using MFM is as follows:

Step 1: Multiply a set of the modulating functions by the differential equation.

Step 2: Integrate the result of Step 1 over an interval period. This step contributes to noise reduction.

Step 3: Apply integration by parts formula to Step 2. This step transfers all the derivatives to a set of modulating functions that we designed to be smooth rather than taking the derivative of noisy measurements.

Step 4: Solve for the unknown parameters or coefficients by taking the inverse of the algebraic linear system.

Remark 1: The number of modulating functions needs to be equal or greater than number of unknown.

The reader can refer to [3] for more details about modulating functions methods.

Next, we provide an adaptive approach of decomposing any signal into basis functions and basis coefficients. Rather than decomposing the signal to predefined basis functions, the signal passes through a learning process where the basis functions will have some features of the decomposed signal.

\section{B. Dictionary Learning Sparse Coding}

The objective of dictionary learning algorithm is to conclude with an updated dictionary $\overline{\mathcal{D}}$. Then when a new signal is provided, the decomposition requires only the new signal and the updated dictionary to solve LASSO (least absolute shrinkage and selection operator) problem for the basis coefficients [9]. The hypothesis of dictionary learning is that the input vector can be decomposed to dictionary and basis coefficients.

$$
S=\overline{\mathcal{D}} \Gamma+\epsilon,
$$

where $S$ is the original input vector, $\Gamma$ is sparse basis coefficients, $\overline{\mathcal{D}}=\left[\bar{d}_{1}(t), \cdots, \bar{d}_{K}(t)\right]$ are basis functions, and $\epsilon$ is the error of reconstruction. The dictionary can be considered as a set of regularly occurring features. By giving training samples

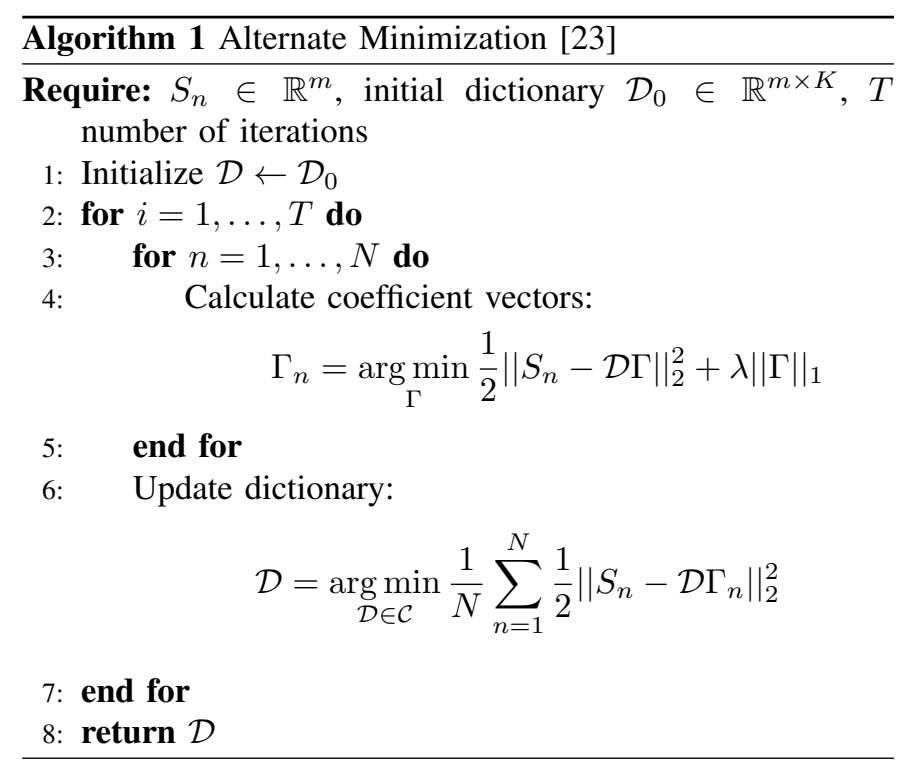

$\left\{S_{n}\right\}_{n=1, \ldots, N}$, the dictionary and basis coefficients are learned simultaneously [18].

$$
\min _{\mathcal{D} \in \mathcal{C}, \Gamma_{n} \in \mathbb{R}^{K}} \frac{1}{N} \sum_{n=1}^{N}\left(\frac{1}{2}\left\|S_{n}-\mathcal{D} \Gamma_{n}\right\|_{2}^{2}+\lambda\left\|\Gamma_{n}\right\|_{0}\right),
$$

where $\mathcal{C}$ is a set of matrices $\mathcal{D}$ in $\mathbb{R}^{m \times K}$ whose columns are the basis vectors $d_{k}=\bar{d}_{k}\left(t_{i}\right)$ with $i=1, \cdots, m$ and have $\ell_{2}$ norm less than or equal to one, $\lambda$ is a regularization parameter, and the input vector $S_{n} \in \mathbb{R}^{m}$. Although, this minimization is not convex because of the $\ell_{0}$-norm. To preserve the convexity, the optimization problem is relaxed to $\ell_{1}$-norm where $\Gamma$ and $\mathcal{D}$ will be obtained alternately see Algorithm 1 .

The initial dictionary $\mathcal{D}_{0}$ in Algorithm 1 can be initialized randomly or taken samples of the original profiles $S$. In Line 3, solving $\Gamma_{n}$ is a well-known optimization problem called basis pursuit denoising [9]. Once $\Gamma_{n}$ is optimized, the dictionary $\mathcal{D}$ will be updated accordingly. The aim is to find an updated dictionary that has the most occurring features stored in it.

Next, we combine both algorithms of MFM and dictionary learning algorithm to provide adaptive estimations.

\section{Combined Dictionary Learning Algorithm and MFM}

In this work, the proposed MFM-DL method aims to improve the estimation by finding numerical basis that passes through a training procedure. In the solar collector, the basis functions or dictionary is trained by a modified version of dictionary learning algorithm, presented in Subsection III-B. Once the dictionary has the most occurring features stored in it, the choice of the basis function in MFM becomes the trained dictionary. In Algorithm 2, $\{S\}_{n=1}^{N} \in \mathbb{R}^{m}$ is the source term profiles. $\mathcal{D}_{0}$ is the initial dictionary where $K$ is the number of features or number of basis functions. In Line 2, the basis coefficients are obtained by solving modulating functions method (see Proposition 1).

Proposition 1: Let the decomposition of the unknown source term $S(t)$ in equation (1) to be $\sum_{k=1}^{K} \alpha_{k} \bar{d}_{k}(t)$, where $\alpha_{k}$ is basis coefficients and $\bar{d}_{k}(t)$ basis functions. And, a set of 


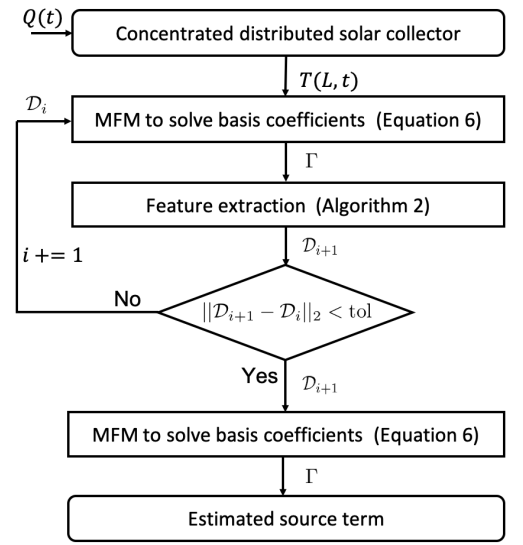

Fig. 2. Training process of the proposed method MFM-DL.

modulating functions $\left\{\phi_{j}(t)\right\}_{j=1}^{j=J}$ that satisfies the properties in Definition 1 , with $J \geq K$. Solving the next algebraic linear system allows to estimate the basis coefficients $\alpha_{k}, k=$ $1, \ldots, K$.

$$
\mathcal{A} \Gamma=\mathcal{K},
$$

where the matrix $\mathcal{A} \in \mathbb{R}^{J \times K}$ has the following form for all components $j=1, \ldots, J$ and $k=1, \ldots, K$.

$$
\mathcal{A}_{j k}=\int_{t_{0}}^{t_{f}} \bar{d}_{k}(t) \phi_{j}(t) d t
$$

and the vector $\mathcal{K} \in \mathbb{R}^{J}$ has the following form for all components $j=1, \ldots, J$.

$$
\mathcal{K}_{j}=\int_{t_{0}}^{t_{f}} u(t) \phi_{j}(t) \frac{\partial}{\partial x} T\left(x^{*}, t\right) d t-\int_{t_{0}}^{t_{f}} \frac{d \phi_{j}(t)}{d t} T\left(x^{*}, t\right) d t,
$$

where $\Gamma \in \mathbb{R}^{K}$ is a vector of the unknowns or basis coefficients $\alpha_{k}$ for all components $k=1, \ldots, K$ and $x^{*}=L$ is a fixed position. The proof of Proposition 1 can be seen in [2].

Once $\Gamma_{n}$ is obtained by MFM, the basis functions will be updated accordingly. When a new profile of the source term is provided, the updated basis functions is used in modulating functions method to solve the basis coefficients. The proposed method is summarized in Fig. 2.

\section{Numerical Simulation Results}

To evaluate the performance of MFM-DL, the method has been numerically examined in open-loop environment. The choice of modulating function are assigned to be polynomialtype since it is simple and smooth. The form of the polynomial-type is given below [3].

$$
\phi_{j}(t)=\left(t_{f}-t\right)^{q+j}\left(t-t_{0}\right)^{q+J+1-j}, \quad t \in\left[t_{0}, t_{f}\right]
$$

where $j=1, \ldots, J$ and $J$ is the number of modulating functions, $q \in \mathbb{R}^{+}$is a degree-of-freedom parameter which tunes the order of modulating functions. Through the entire simulation, the parameter $q$ is fixed to 3 . The model's parameters of the concentrated distributed solar collector are chosen to be $\rho=903, c=1820, A_{s}=0.0006, v=0.73, G=1.83$, and $L=172$ according to [7]. The proposed method has been tested by considering two scenarios, which are synthetic and real solar irrandiance.
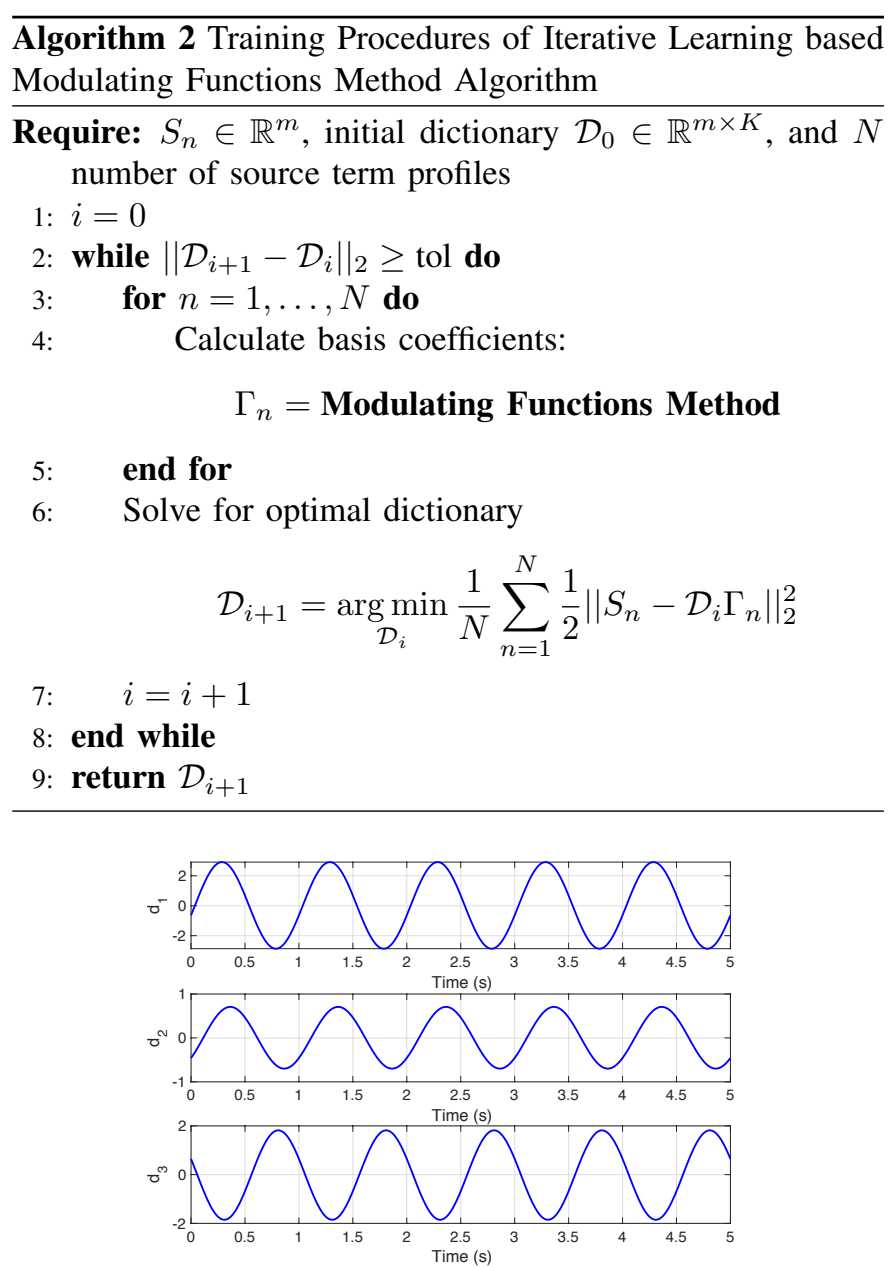

Fig. 3. Plotting the columns of the updated dictionary after the training procedure in Algorithm 2.

\section{A. Synthetic Solar Irradiance}

The first numerical test aims to find an estimation of the source term $S(t)$, which depends on the solar irradiance $I(t)$. The time-varying profile of the solar irradiance is chosen to have sinusoidal form. The volumetric flow rate $Q(t)$ varies from $0.001 \mathrm{~m}^{3} / \mathrm{s}$ to $0.012 \mathrm{~m}^{3} / \mathrm{s}$ due to the pump's physical limitation. In Algorithm 2, the dictionary $\mathcal{D}_{0}$ is initialized randomly and each column of the dictionary has $\ell_{2}$-norm less than one. The number of the training source term profile is $N=4$ and the number of columns of the dictionary is $K=3$. The resultant dictionary after the training procedure is illustrated in Fig. 3 where updated dictionary $\mathcal{D}=\left[d_{1}, d_{2}, d_{3}\right]$. The estimation result using the updated $\mathcal{D}$ is shown in Fig.4. The relative errors are in Table II.

The training procedure's objective is to find numerical basis functions that contain the most occurring features in it. Next is to validate the updated basis functions by testing it with a new signal that has not been provided in the training procedure. This estimation uses only the modulating functions methods by providing the updated basis functions or dictionary. To improve the estimation, the new signal should have the same form as in the training procedure. The result of the source term estimation appears in Fig. 5. Whereas, the relative error of estimation is $\frac{\|S-\hat{S}\|_{2}}{\|S\|_{2}} \times 100=0.0489 \%$. 

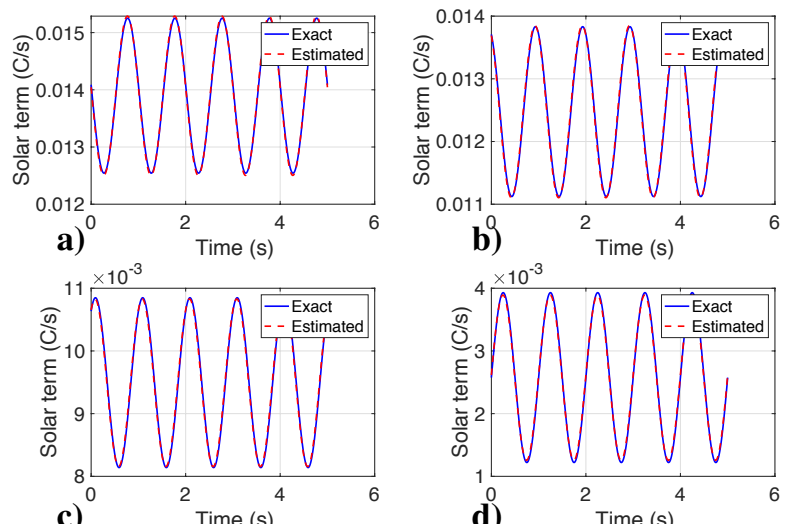

Fig. 4. Plotting the exact and the estimated source terms that have been considered in the training procedure using synthetic solar irradiance.

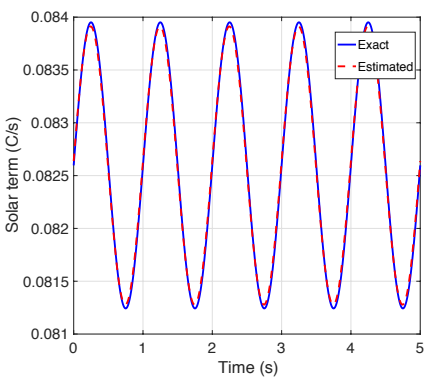

Fig. 5. Plotting the exact and the estimated source terms by providing a new synthetic solar irradiance.

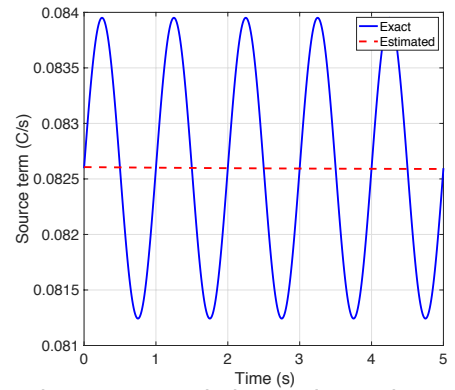

Fig. 6. Plotting the exact and the estimated source terms by the standard MFM with polynomial basis.

To compare the proposed method to standard MFM with predefined basis functions, Fig. 6 shows the estimation of the source term using polynomial basis functions. The number of the basis functions is fixed to $K=3$ to compare both methods fairly. The estimation of using predefined basis does not perform well due to the choice of the basis functions. Since the source term in this simulation is known to have sinusoidal form, it is better to choose Fourier basis functions than polynomial basis functions. Alternatively, increasing the number of the polynomial basis $K$ can also result in good estimation. Beside the type of the predefined basis functions, the number of the basis functions is important as this may lead to bad estimation in case few basis functions and numerical instabilities in case of using a big number of basis functions.

\section{B. Real Solar Irradiance Profile}

This numerical test considers real profiles of solar irradiance. The objective is to train the basis functions by
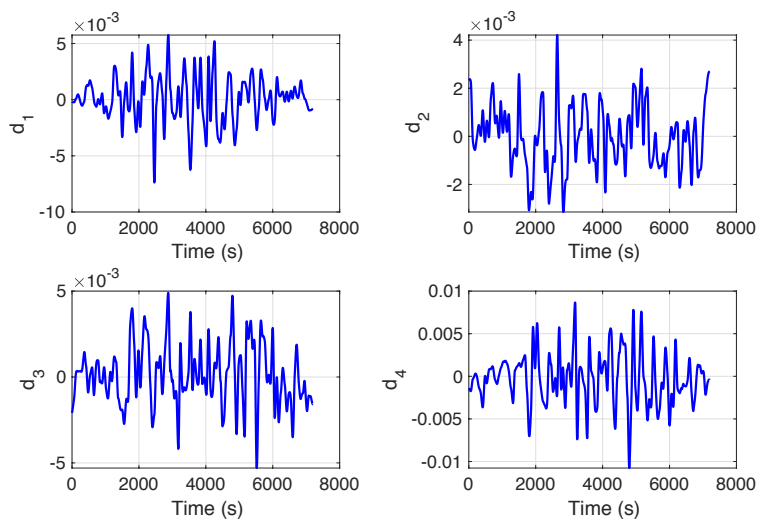

Fig. 7. Plotting four columns of the updated dictionary after the training procedure in Algorithm 2
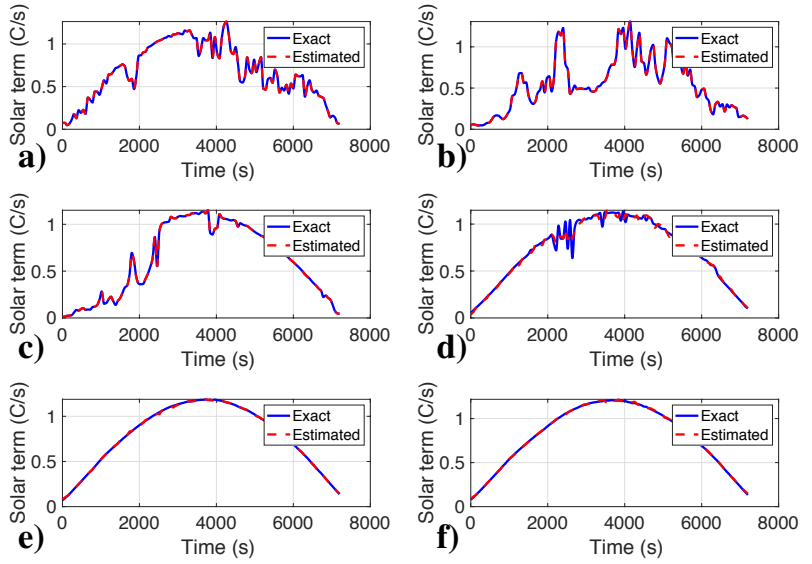

Fig. 8. Plotting six samples of the exact and the estimated source terms that have been considered in the training procedure using real solar irradiance.

providing real solar irradiance profiles and find estimation of the source term $S(t)$ that has not been considered in the training procedure. The solar irradiance profiles used in this numerical test was recorded in Solar Village, Saudi Arabia [1]. The training set consists of $N=50$ solar irradiance profiles from February 1, 2002 to March 23, 2002. The dictionary is initialized randomly and each column has $\ell_{2}$-norm less than one where $K=28$. And, $Q(t)$ varies from $0.001 \mathrm{~m}^{3} / \mathrm{s}$ to $0.012 \mathrm{~m}^{3} / \mathrm{s}$. Four samples of updated dictionary is shown in Fig. 7 which can be seen clearly that the dictionary does not have the characteristic of the predefined basis functions. Furthermore, Fig. 8 shows six samples of the estimated source terms using the updated dictionary $\mathcal{D}$ from February 1 to February 6, 2002. The relative errors are illustrated in Table II.

Once the dictionary is trained, the objective is to use the trained dictionary along with modulating functions methods to find an estimation of the source term. Fig. 9 shows the source term estimation where the solar irradiance profile is considered in March 30, 2002. The relative error of estimation is $\frac{\|S-\hat{S}\|_{2}}{\|S\|_{2}} \times 100=1.8387 \%$. Fig. 10 shows the difference error between the exact and estimated source term.

\section{CONCLUSION}

In this work, an iterative learning based on modulating functions method is designed for adaptive estimation of the source 
TABLE II

RELATIVE ERROR ESTIMATION OF THE TRAINED DICTIONARY USING SYNTHETIC AND REAL SOLAR IRRADIANCE PROFILES

\begin{tabular}{|c||cccccc|}
\hline Samples & a) & b) & c) & d) & e) & f) \\
\hline \hline Synthetic data & $0.3065 \%$ & $0.4416 \%$ & $0.5703 \%$ & $1.4694 \%$ & -- & -- \\
\hline Real data & $0.1387 \%$ & $0.3227 \%$ & $0.1232 \%$ & $3.9773 \%$ & $0.5446 \%$ & $0.6225 \%$ \\
\hline
\end{tabular}

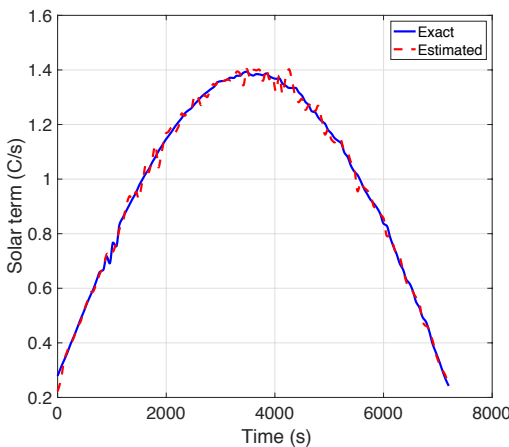

Fig. 9. Plotting the exact and the estimated source terms by providing a new real solar irradiance profile.

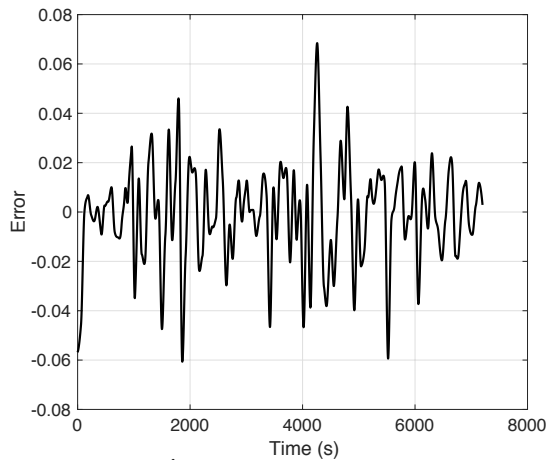

Fig. 10. Estimation error $\hat{\boldsymbol{S}}(t)-\boldsymbol{S}(\boldsymbol{t})$ using the proposed method.

term. The concept of using modulating functions method with predefined basis functions may provide inaccurate estimation depending on the selection of predefined basis. The proposed method (MFM-DL) is designed to capture the estimated source term features, which can then be used as adaptive basis functions that improve estimation. Numerical simulations tested the proposed method in two cases, synthetic and real solar irradiance profiles.

In future work, the proposed method will be extended to an online estimation where the training procedure of the basis functions will be updated along with predicting the source term. Besides the online approach, the proposed method will consider a new case estimation when the source term is time and space varying $\hat{S}(x, t)$. Rather than estimation the source term at the boundary, the proposed method may ease the estimation.

\section{REFERENCES}

[1] NASA Remote Sensing Validation Data: Solar Village, Saudi Arabia (1998-2002).

[2] S. Asiri, S. Elmetennani, and T.-M. Laleg-Kirati. Moving-Horizon Modulating Functions-Based Algorithm for Online Source Estimation in a First Order Hyperbolic PDE. Journal of Solar Energy Engineering, 2016.
[3] S. Asiri and T. M. Laleg-Kirati. Modulating functions-based method for parameters and source estimation in one-dimensional partial differential equations. Inverse Problems in Science and Engineering, 25(8):11911215 , aug 2017.

[4] S. Asiri, D. Liu, and T.-M. Laleg-Kirati. Selection of modulating functions' design parameters for estimation problems. IEEE Control Systems Letters, 5(1):277-282, 2021.

[5] S. Asiri, C. Zayane, and T.-M. Laleg-Kirati. An adaptive observer-based algorithm for solving inverse source problem for the wave equation. Mathematical Problems in Engineering, 2015:1-8, 102015.

[6] Z. Belkhatir and T. M. Laleg-Kirati. Parameters and fractional differentiation orders estimation for linear continuous-time non-commensurate fractional order systems. Systems Control Letters, 115:26 - 33, 2018.

[7] F. E. Camacho, M. M. M. Berenguel, and F. R. F. R. Rubio. Advanced Control of Solar Plants. Springer, 1997.

[8] F. E. Camacho, R. F. Rubio, M. Berenguel, and L. Valenzuela. A survey on control schemes for distributed solar collector fields. part i: Modeling and basic control approaches. 2007.

[9] S. S. Chen, D. L. Donoho, and M. A. Saunders. Atomic decomposition by basis pursuit. SIAM J. Sci. Computer, 20:33-61, 1998.

[10] F. Fischer and J. Deutscher. Flatness-based algebraic fault diagnosis for distributed-parameter systems. Automatica, 117, 042020.

[11] F. Fischer, J. Deutscher, and T.-M. Laleg-Kirati. Source estimation for first order time-varying hyperbolic systems. 072018.

[12] A. Gallego and E. Camacho. Estimation of effective solar irradiation using an unscented kalman filter in a parabolic-trough field. Solar Energy, 86(12):3512 - 3518, 2012. Solar Resources.

[13] T. A. Johansen and C. Storaa. Energy-based control of a distributed solar collector field. Automatica, 38(7):1191-1199, jul 2002.

[14] D. Liu, T. Laleg-Kirati, O. Gibaru, and W. Perruquetti. Identification of fractional order systems using modulating functions method. In 2013 American Control Conference, pages 1679-1684, 2013.

[15] D. Liu, T. Laleg-Kirati, W. Perruquetti, and O. Gibaru. Non-asymptotic state estimation for a class of linear time-varying systems with unknown inputs. IFAC Proceedings Volumes, 47(3):3732 - 3738, 2014. 19th IFAC World Congress.

[16] D.-Y. Liu and T.-M. Laleg-Kirati. Robust fractional order differentiators using generalized modulating functions method. Signal Processing, 107:395 - 406, 2015. Special Issue on ad hoc microphone arrays and wireless acoustic sensor networks Special Issue on Fractional Signal Processing and Applications.

[17] W. Muniz, F. Ramos, and H. de Campos Velho. Entropy- and tikhonovbased regularization techniques applied to the backwards heat equation. Computers Mathematics with Applications, 40(8):1071 - 1084, 2000.

[18] B. A. Olshausen and D. J. Field. Sparse coding with an overcomplete basis set: A strategy employed by V1? Vision Research, 37(23):3311$3325,1997$.

[19] F. Perdreauville and R. Goodson. Identification of systems described by partial differential equations. ASME J. Fluids Eng., 88(2):463-463, 1966.

[20] H. Preisig and D. Rippin. Theory and application of the modulating function method-I. review and theory of the method and theory of the spline-type modulating functions. Comput. Chem. Eng., 17(1):1-16, 1993.

[21] M. Shinbrot. On the Analysis of Linear and Nonlinear Dynamical Systems From Transient-Response Data. 1954.

[22] R. Silva, L. Rato, and J. Lemos. Time scaling internal state predictive control of a solar plant. Control Engineering Practice, 11(12):14591467, 2003.

[23] B. M. Whitaker and D. V. Anderson. Learning anomalous features via sparse coding using matrix norms. In 2015 IEEE Signal Processing and Signal Processing Education Workshop, SP/SPE 2015, pages 196-201. Institute of Electrical and Electronics Engineers Inc., dec 2015. 